\title{
Expansion capacity of bran-enriched doughs in different scales of laboratory mixers
}

Link to publication record in Manchester Research Explorer

\section{Citation for published version (APA):}

Campbell, G., Campbell, GM., Ross, M., Motoi, L., Campbell, G. M. (Ed.), Scanlon, M. G. (Ed.), \& Pyle, D. L. (Ed.) (2008). Expansion capacity of bran-enriched doughs in different scales of laboratory mixers. In G. M. Campbell, M. G. Scanlon, \& D. L. Pyle (Eds.), Bubbles in Food 2: Novelty, Health and Luxury (pp. 337-354). Eagan Press.

\section{Published in:}

Bubbles in Food 2: Novelty, Health and Luxury

\section{Citing this paper}

Please note that where the full-text provided on Manchester Research Explorer is the Author Accepted Manuscript or Proof version this may differ from the final Published version. If citing, it is advised that you check and use the publisher's definitive version.

\section{General rights}

Copyright and moral rights for the publications made accessible in the Research Explorer are retained by the authors and/or other copyright owners and it is a condition of accessing publications that users recognise and abide by the legal requirements associated with these rights.

\section{Takedown policy}

If you believe that this document breaches copyright please refer to the University of Manchester's Takedown Procedures [http://man.ac.uk/04Y6Bo] or contact uml.scholarlycommunications@manchester.ac.uk providing relevant details, so we can investigate your claim.

\section{OPEN ACCESS}




\title{
Chapter 32
}

\section{Expansion Capacity of Bran-Enriched Doughs in Different Scales of Laboratory Mixers}

\author{
Grant M Campbell ${ }^{1}$, Marcela Ross ${ }^{2}$ and Lidia Motoi $^{2}$ \\ ${ }^{1}$ Satake Centre for Grain Process Engineering, School of Chemical Engineering \\ and Analytical Science, The University of Manchester, Manchester, M60 1QD, UK \\ ${ }^{2}$ New Zealand Institute for Crop \& Food Research Limited, Lincoln, New Zealand
}

\begin{abstract}
The dynamic dough density system, which simulates growth during proving of bread dough, was refined to enhance the accuracy and reproducibility of the technique. The system was then used to quantify the expansion capacity of doughs, as indicated by the minimum density achieved by a proving dough piece, in three different scales of geometrically similar laboratory dough mixers. The effects of bran level on dough expansion and baked loaf characteristics were investigated. Dynamic dough density measurements showed no difference in expansion capacity during proving from doughs mixed in the three scales of mixer. Bran at $7.5 \%$ flour substitution had no significant effect on the minimum density, but decreased loaf volumes compared with no bran. Addition of bran at $15 \%$ substitution decreased loaf volume further and also decreased the maximum expansion during proving, indicating that at this level bran exerts at least part of its effect in reducing loaf volumes during the proving stage of the breadmaking process. Baking scores were comparable for loaves baked from doughs mixed in the two larger scales of mixer, while specific loaf volume decreased as mixer scale decreased.
\end{abstract}

Keywords: Bread dough; bran; proving; proof; mixer design; mixer scale; mass transfer; carbon dioxide; yeast fermentation

Abbreviations: DDD, dynamic dough density; MDD, mechanical dough development.

\section{Introduction}

Wheat flour doughs are unique in their ability to retain fermentation gases and expand to produce an aerated structure that can be baked into a palatable loaf (He and Hoseney, 1991). This ability arises from the viscoelastic and strain hardening properties of the gluten proteins in the dough (Dobraszczyk and Morgenstern, 2003). The inherent ability of wheat gluten to form a gas-retaining network can be manipulated in order to achieve baked loaves of distinctive structure and texture, by modifying the dough formulation with respect to water level and added ingredients. In mechanical dough development (MDD) processes, the amount and rate of work imparted to the dough also determines its ability to retain gases during fermentation and baking (Heaps et al., 1967; Chamberlain et al., 1967; Kilborn and Tipples, 1972; Frazier et al., 1975; Skeggs and Kingswood, 1981; Wilson et al., 2001). 


\section{4 / Chapter 32}

The dynamic dough density (DDD) technique introduced by Campbell et al. (2001), and described in further detail by Chiotellis and Campbell (2003b), allows the changing density of a fermenting dough piece to be monitored continuously, thereby indicating the rate of mass transfer of carbon dioxide gas into bubbles. By allowing the sample to continue to grow beyond the times reported by Campbell et al. (2001), the maximum capacity of the dough to expand and retain gas can also be quantified as the minimum density achieved. The minimum density is attained when the carbon dioxide gas is lost from the system at the same rate that it is produced by the yeast. Gas is lost through diffusion from the surface of the growing dough piece and through rupture of stretched gluten membranes. Different dough ingredients can potentially affect the rate of loss of gas by affecting either or both of these mechanisms. Some ingredients may exert their influence principally during the proving stage of breadmaking, while others only affect the dough gas retention capacity during baking. The DDD system allows the effect of the former group of dough components to be identified and monitored. However previous work has not extended the technique to identify the point of minimum density.

Bran is one of many ingredients that can be added to dough formulations, and an important one from the perspective of manufacturing healthy bakery goods. The addition of bran to dough formulations does not decrease the gassing power of the dough, but decreases gas retention (Pomeranz et al., 1977; Sosulski and Wu, 1988). Depending on the nature of the bran, the dough formulation and the breadmaking system, at least some of this effect is likely to occur during proving, such that the ability of the dough to retain gas during proving is compromised. The DDD technique can potentially throw light on the action of bran during breadmaking; this paper reports some preliminary investigations into the potential of the refined technique for studying bran effects.

Most research investigations of breadmaking systems, dough formulations and suchlike are carried out in laboratory-scale mixers, but few studies of the effects of mixer scale-up have been reported (Martin et al., 2004), in part because few bakery research laboratories have access to several scales of geometrically similar mixers. Several research laboratories have scaled down the breadmaking process, to allow studies when only small amounts of sample are available, using, for example, thimbles to bake loaves (Gras and Békés, 1996; Békés and Wrigley, 2002). Bran particle size is an important factor affecting its functionality in breadmaking (Shetlar and Lyman, 1944; Pomeranz et al., 1977; Zhang and Moore, 1997, 1999; de Kock et al., 1999), but these small loaves have length scales only a few times the dimension of a typical bran particle, such that conclusions drawn from them may not scale well. The current work therefore took the opportunity to investigate the effects of scale in three geometrically similar laboratory scale MDD mixers with respect to the consequences of bran addition.

The objectives of the current work were: to refine the DDD system to improve accuracy and assess reproducibility; to use the system to investigate the bubble growth dynamics of doughs mixed in three scales of mixer and relate these to baked loaves; and to investigate the effect of bran on dough expansion and on baked loaves from three scales of mixer.

\section{Materials and Methods \\ 2.1. Dough preparation}

Doughs were prepared in three scales of mechanical dough development mixer, based on 10 , 50 and $125 \mathrm{~g}$ of flour, respectively. The MDD125 mixer has been described previously by Wilson $e t$ al. $(1997,2001)$, and comprises a bowl containing two vertically mounted and counter-rotating flatbladed paddles, $58 \mathrm{~mm}$ in diameter, rotating at $138 \mathrm{rpm}$ and with a close clearance with the walls of the bowl. The MDD50 and MDD10 mixers are scaled-down versions of the $125 \mathrm{~g}$ mixer, with paddles of 44 and $23 \mathrm{~mm}$, rotating at 130 and $140 \mathrm{rpm}$, respectively. The recipe consisted of (flour + bran) 100\%; water (variable); yeast 3\% for baking trials, $6 \%$ for DDD trials; salt 2\%; WRIfat $1.2 \%$ (comprising 20\% SSL, 15\% DATEM, 20\% enzyme-active soya flour and the remainder flour and anticaking agent); sugar $0.75 \%$; bromate 50 ppm; ascorbic acid 100 ppm. The flour was Crop \& 
Food Research's (C\&FR) standard long-term test baking flour (a medium quality flour supplied by Weston Milling, Christchurch, New Zealand) with 11.2\% protein, $15.1 \%$ moisture, 0.9 colour grade, 59.8\% Farinograph water absorption (as determined according to C\&FR's in-house methods T7, T2, T5 and A8, respectively). Either $0,7.5$ or $15 \%$ of the flour weight was replaced with commercial bran (Flemings Bran Flakes, manufactured by Champion Flour Mills, Christchurch, New Zealand). The optimum work input requirements and water absorption were determined in the $125 \mathrm{~g}$ mixer (according to C\&FR's in-house method B7) for each bran level and used in all three mixers.

For baking trials, the entire dough from each mix was recovered and baked according to C\&FR's standard in-house methods (these vary slightly for the different scales of mixer because the smaller loaves heat or cool more rapidly). For the $125 \mathrm{~g}$ mixer, doughs were given 10 minutes first proof at $32^{\circ} \mathrm{C}$, moulded through a Mono bench top moulder, given a final proof for 45 minutes at $40^{\circ} \mathrm{C}$ and $80-90 \%$ relative humidity, and baked at $220^{\circ} \mathrm{C}$ for 25 mins. For the $50 \mathrm{~g}$ mixer, first proof was for 8 minutes, after which doughs were moulded in a Hunt's bun moulder, and after 45 minutes final proof were baked at $208^{\circ} \mathrm{C}$. For the $10 \mathrm{~g}$ mixer, loaves were prepared as described by Wooding (1997). First proof was for 8 minutes at $39.5^{\circ} \mathrm{C}$. The dough was then sheeted through a domestic pastry sheeter with a roll gap of $2.5 \mathrm{~mm}$ and rolled manually under a pressure board with a gap of $13 \mathrm{~mm}$. The dough was placed in a baking pan and given a second proof for 8 minutes at $39.5^{\circ} \mathrm{C}$, to recover from cooling during the sheeting and moulding process, before final proof at $40^{\circ} \mathrm{C}$ for 22 minutes and baking at $208^{\circ} \mathrm{C}$ for 13 minutes. Baked loaves were cooled overnight and weight and volume measured the following day (by rapeseed displacement for the loaves from the MDD125 and MDD50, and by displacement of $0.4 \mathrm{~mm}$ glass beads for the MDD10 loaves), and texture evaluated on a 1-11 scale by an experienced baker. The baking score was calculated for the loaves from the two larger mixers as (arbitrary loaf volume score + texture score), where arbitrary loaf volume was calculated as (volume in $\mathrm{cm}^{3}-x$ ) $/ y$, where $x$ was 540 and 230, and $y$ was 18 and 5.7, for loaves from the MDD125 and MDD50, respectively. (Arbitrary loaf volume is simply an expedient way of converting loaf volume to a numerical score of comparable magnitude to the texture score, such that the two added together give a single quantitative measure of loaf quality that combines attributes of both volume and texture. A corresponding equation for calculating the arbitrary loaf volume appropriate for loaves from the MDD10 was not available.)

\subsection{Dynamic dough density}

The DDD system was based on a double cup as described by Campbell et al. (2001) and Chiotellis and Campbell (2003b), in which a dough sample was initially weighed in air and then immersed in xylene and weighed again. From the difference in weights the density at time $t, \rho(t)$, was calculated as:

$$
\rho(t)=\frac{m_{\text {air }}}{m_{\text {air }}-m_{x y l e n e}} \rho_{x y l e n e}
$$

where $m_{\text {air }}$ and $m_{x y l e n e}$ are the recorded weights in air and immersed in xylene, respectively, and $\rho_{x y l e n e}$ is the density of xylene $\left(0.85 \mathrm{~g} \mathrm{~cm}^{-3}\right.$ at $\left.40^{\circ} \mathrm{C}\right)$. The xylene was maintained at $40^{\circ} \mathrm{C}$ by circulating water from a water bath through a jacketed beaker. An anti-float mesh was constructed to catch samples once they began to float; it was made slightly larger than the lower cup and was gently rounded to avoid excessive local strains on the sample. The system used a Mettler AE200 balance, accurate to $0.1 \mathrm{mg}$, to record the initial and immersed weights of the dough sample. A second system was built, to allow a greater number of samples to be processed within a day; this system used a less precise Mettler PB153-S balance accurate to $1 \mathrm{mg}$. Both systems were operated in a fume cupboard for safety. Unless otherwise stated, all reported measurements were made using the first system. Weights were logged every $10 \mathrm{~s}$ to a laptop computer using Labview 6.1 software (National Instruments, Austin, Texas, USA). All times are taken as the time from the end of mixing; typically it took about 2-3 minutes to retrieve a sample and begin monitoring its changing density. 


\subsection{Optimisation of sampling protocol}

Four sampling procedures were evaluated, using standard dough formulations without bran as described above. In the first, a sample of about 4-6 g in weight was cut from the dough piece using a sharp razor blade. In the second procedure, a sample of about $10 \mathrm{~g}$ was cut from the dough piece, rolled into a cylinder between two Perspex sheets held $13 \mathrm{~mm}$ apart, a section of about $5 \mathrm{~g}$ in weight cut from the middle of this, and this small section turned through $90^{\circ}$ and again rolled to give a squat cylindrical shape. In the third procedure, the dough piece ex-mixer was gently sheeted, using a rolling pin and some rectangular Perspex rods, to a thickness of $12 \mathrm{~mm}$, from which a sample was taken using a $21 \mathrm{~mm}$ diameter metal cookie cutter, and gently swirled in a spherical flask to strengthen the outer surface. In the fourth procedure, the dough piece ex-mixer was allowed to rest for 7 minutes at $32^{\circ} \mathrm{C}$ before sampling as described in Procedure 3 .

\subsection{Evaluation of accuracy and reproducibility}

To evaluate the accuracy and reproducibility of the technique, doughs without bran were mixed in duplicate to different work inputs, and the dough density profile and minimum density recorded. To establish the effect of using twice the normal quantity of yeast, doughs without bran were mixed in duplicate with $3,4.5$ and $6 \%$ yeast on flour weight. To compare the two DDD systems, doughs were mixed with $0,7.5$ and $15 \%$ bran substitution and duplicate samples from the same dough run in parallel through the two systems.

\subsection{Effects of mixer scale and bran level on dough expansion capacity and baked loaf specific volumes}

Dough formulations with $0,7.5$ and $15 \%$ bran substitution were mixed in duplicate in each mixer, with double the normal yeast quantity, and sampled for DDD measurements. Separate doughs for baking trials were also mixed in duplicate in each mixer, with the normal yeast level (3\% on flour weight). All trials, both the DDD and the baking trials, were performed in a random order within a single day. For the DDD trials, both systems were used, with the samples for each experimental condition run in a random order through one system and independently in the reverse order through the other.

Presoaking of bran affects water absorption and baked loaf volume, due to hydration effects and possibly activation of indigenous lipoxygenase (Dreese and Hoseney, 1982; Sosulski and Wu, 1988; Lai et al., 1989a,b; Nelles et al., 1998). In a separate trial, the effects of presoaking the bran in its own weight of water on the optimum work input and water absorption level and on the baked loaf volume were investigated. Presoaking for 4 or 6 hours increased optimum work input and water absorption compared with untreated bran, and improved loaf volume and texture, in general agreement with the work referenced above. However, in order to magnify the effects of bran on baked loaves, presoaking of the bran was not used in the other trials described in this paper. For the same reason, the dough formulations with bran were not supplemented with vital wheat gluten, as is sometimes practised to offset the dilution effect of the bran (Pomeranz, 1977; Dubois, 1978; Shogren et al., 1981; Lai et al., 1989c; Gan et al., 1989, 1992).

\section{Results and Discussion}

\subsection{Sampling protocol}

The manner in which samples for DDD measurements are taken from doughs greatly influences the density profile obtained. In particular, it is easy through sampling to introduce points of weakness in the surface of the dough sample from which gas preferentially and unrepresentatively escapes, giving a characteristic "hiccup" in the density profile that indicates a sudden loss of gas. The frequency of the occurrence of these points of weakness and the resulting hiccups is random, 
with certain dough formulations or preparation conditions increasing the likelihood that a point of weakness will occur. Thus, Sampling Protocol 1, in which samples were cut from the dough with a sharp razor, was prone to these random points of weakness, such that results using this sampling protocol were variable and unreliable. This approach also gave wide variations in the weights of samples obtained; widely varying sample sizes could affect the relative rates of mass transfer of $\mathrm{CO}_{2}$ into bubbles and from the surface of the dough, so it is desirable to minimise sample weight variation. This method of sampling is adequate for measuring the density of (yeasted or unyeasted) doughs immediately $e x$-mixer, but is not suitable for obtaining samples that will subsequently be allowed to expand to their maximum capacity in the DDD system.

The remaining three sampling protocols investigated were designed to alleviate or eliminate these points of weakness. Protocol 2, rolling a $10 \mathrm{~g}$ sample into a cylinder, removing a $5 \mathrm{~g}$ sample from the centre of the cylinder and rerolling the sample, tended to eliminate the hiccups but gave a very stable sample, giving rise to concerns that the rolling process itself was developing the dough rheology to an extent that could mask inherent responses to experimental variables. Protocol 3 gave relatively uniform sample weights, improving the reproducibility of the technique by avoiding large differences in surface:volume ratio, and the gentle swirling procedure strengthened the surface of the dough piece such that random points of weakness were largely eliminated. Protocol 4, in which the sample was obtained after a rest period, gave a very stable sample that took an excessively long time to reach the minimum density. In all cases the time required to retrieve the sample and begin monitoring its density could affect the results, due to cooling and possibly drying of the samples, so it is desirable to minimise this time and to keep it as consistent as possible. On the basis of these trials, Protocol 3, removing a sample from a gently sheeted dough using a circular cookie cutter and gently swirling the sample in a spherical flask, was adopted for the rest of the work reported here; this technique gave samples with weights generally in the range 4-5 g and with a low variation.

Figure 1 illustrates typical density profiles obtained from the four sampling techniques, using a lean dough formulation with no added emulsifier (WRIfat). The different starting times of the density profiles reflect the relative times required to retrieve samples using the different protocols. In this case the dough was unstable, and sampling Protocols 1, 2 and 3 demonstrated this instability

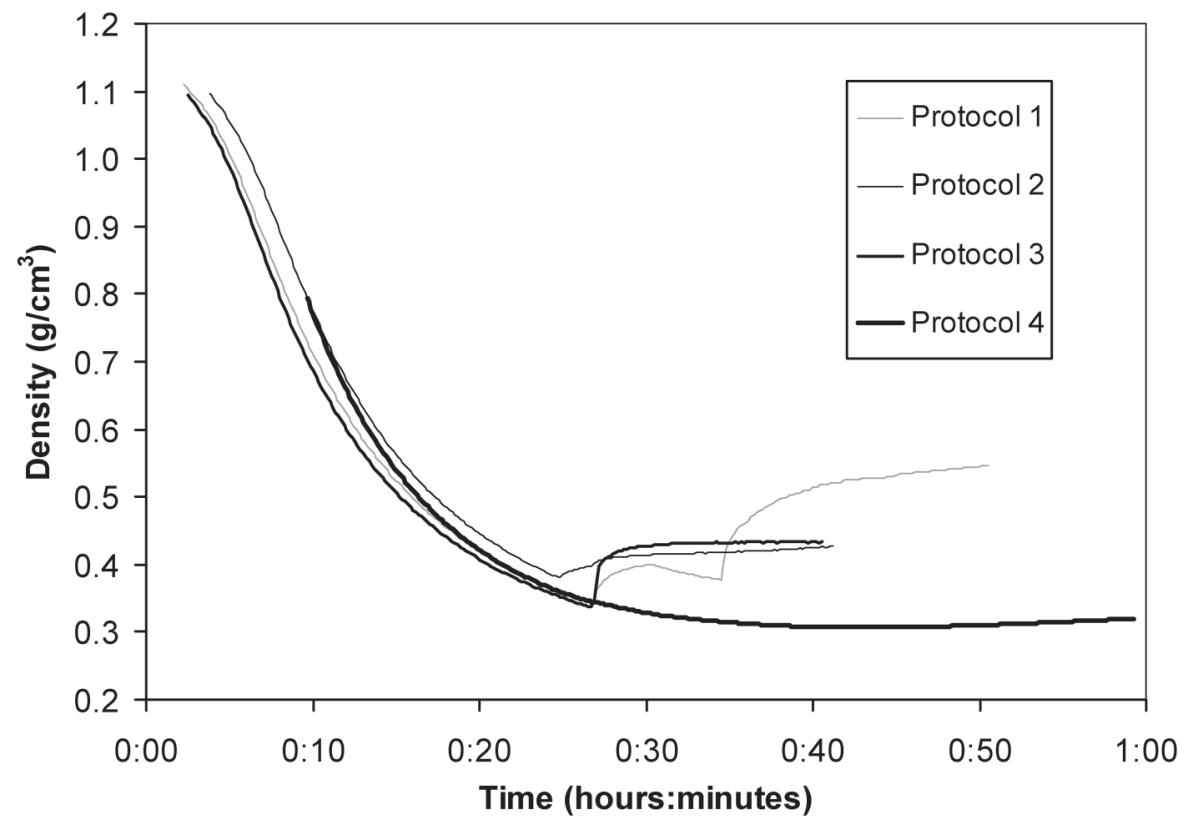

Figure 1. Typical density profiles obtained using different sampling protocols, for a dough formulation with no emulsifier and no bran. 


\section{8 / Chapter 32}

to differing extents, while Protocol 4 masked the instability. The stability of the dough following the rest period in Protocol 4 reflects the bakery practice of allowing a period of intermediate proof following mixing to strengthen and restore the dough. These results illustrate how Protocol 3 preserves the instability of inherently weak samples, but does not exacerbate inherent instability by encouraging points of weakness.

\subsection{Accuracy and reproducibility}

The minimum density achieved, $\rho_{\min }$, was taken as indicative of the maximum expansion capacity of a dough. Figure 2 shows the minimum density obtained from duplicate doughs mixed in the MDD125 in a random order to different work inputs. (N.B. The scale in this and similar graphs has been kept consistent to aid comparison. Error bars are in all cases \pm 1 standard deviation; where not shown, they are smaller than the symbols used on the graph.) Clearly the reproducibility of the technique was good, and the technique was able to distinguish the effect of a processing variable (work input) on expansion capacity. A pooled standard deviation was calculated from these data to be $0.0014 \mathrm{~g} \mathrm{~cm}^{-3}$. The optimum work input for this dough, based on torque profile during mixing, was determined to be $12.4 \mathrm{Wh} \mathrm{kg}^{-1}$, which did not correspond with the work input that produced the minimum density. Loaves were not baked in this work (as its purpose was solely to evaluate the accuracy and reproducibility of the DDD technique), but it would have been interesting to see how work input and minimum density correlated with baked loaf volume and texture.

The minimum density obtained from duplicate doughs mixed with $3,4.5$ and $6 \%$ yeast showed no significant difference $(\mathrm{P}=0.29)$. Treating the six data points as replicates gave a standard deviation of $0.0080 \mathrm{~g} \mathrm{~cm}^{-3}$, somewhat larger than from the previous trials. Using double the standard yeast quantity caused the minimum density to be reached after about 40 minutes, compared with 52 minutes at $4.5 \%$ and 101 minutes with just 3\% yeast; the effect of yeast level on the time

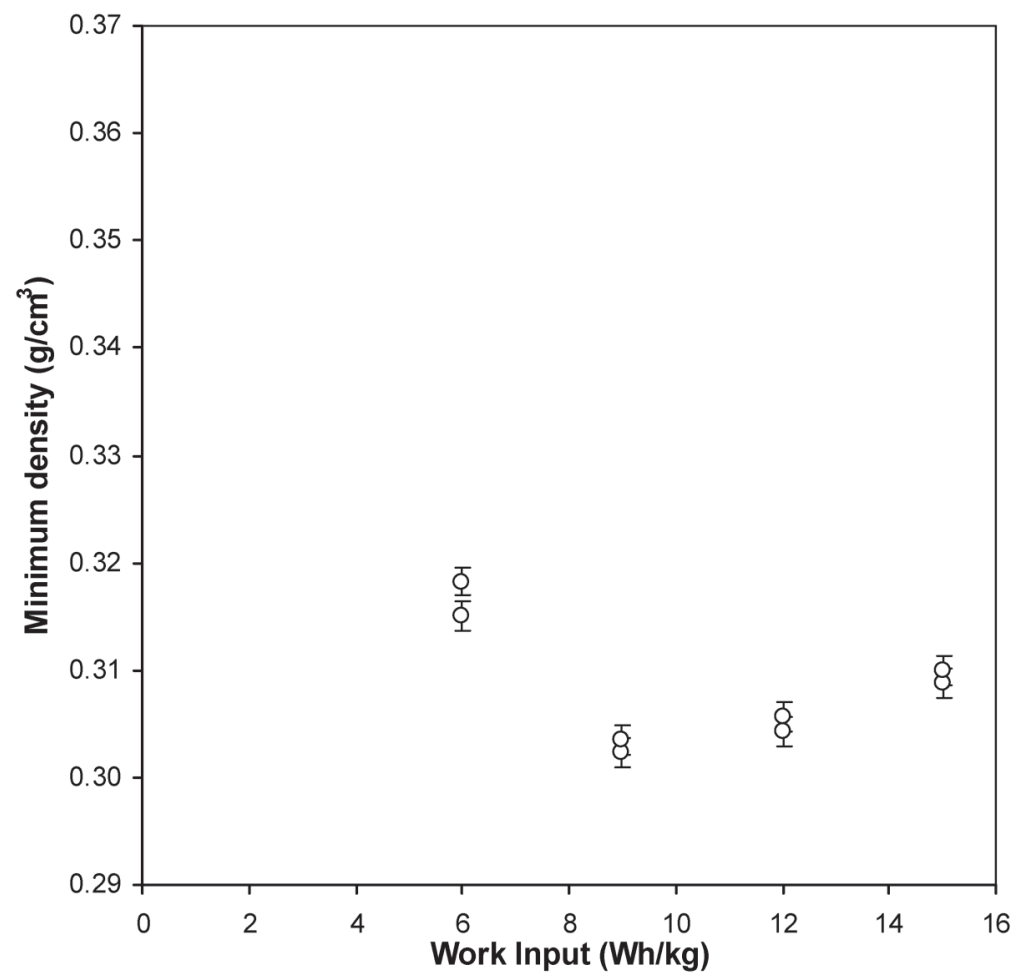

Figure 2. Effect of work input on the maximum expansion capacity (minimum density) of doughs mixed in the MDD125 mixer. 
appeared not to be directly inversely proportional. A 40 minute run time plus sample preparation time was sufficiently practical to allow a reasonable number of trials to be performed within a day, particularly with two density meters available.

Figure 3(a) shows the density profiles obtained from the two density meters for doughs containing $0,7.5$ and $15 \%$ bran. Clearly the profiles from the two systems were comparable, as indicated also by the minimum densities shown in Figure 3(b), from which the pooled standard
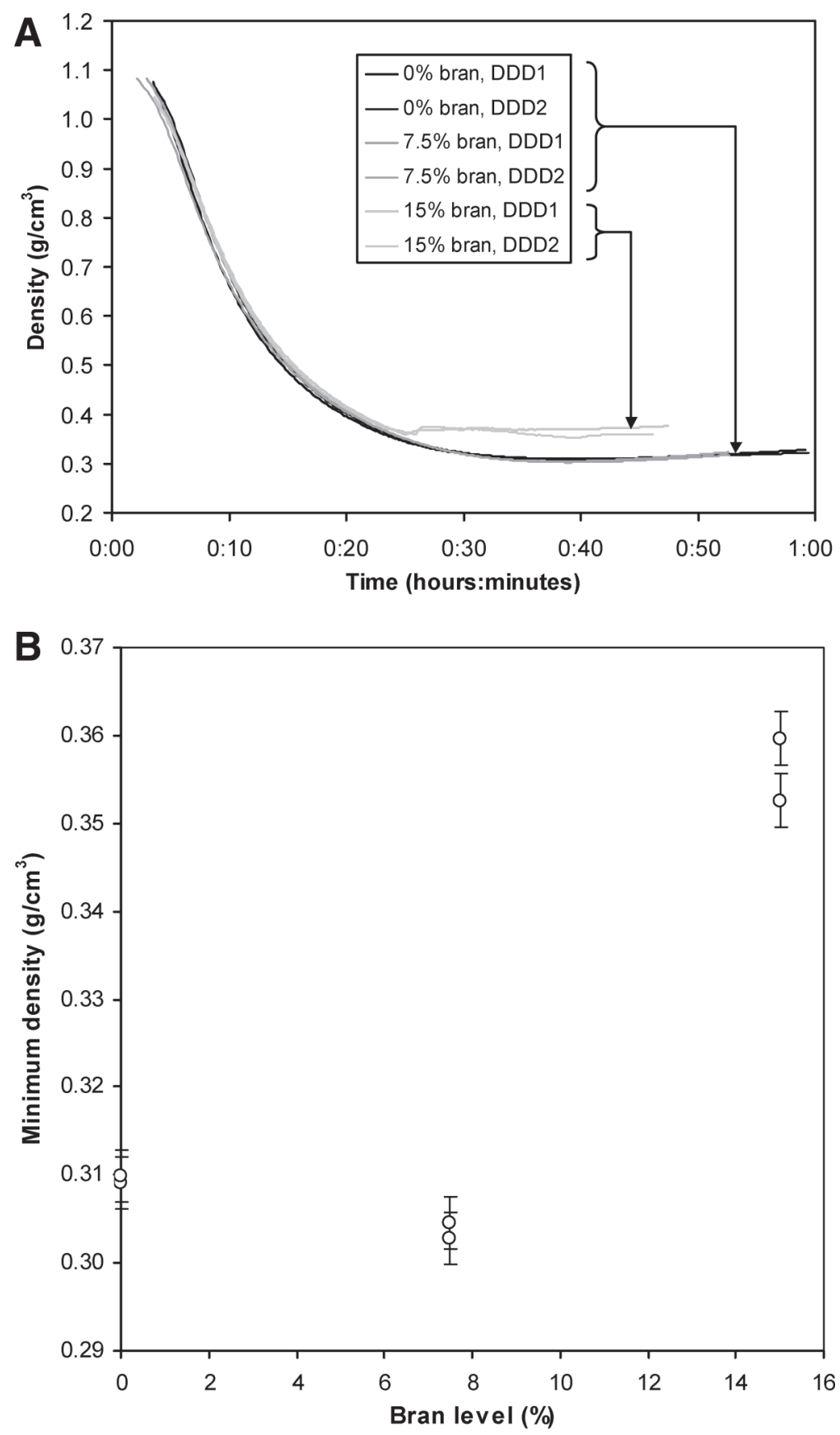

Figure 3. Effect of bran level on (a) density profiles and (b) minimum density from doughs containing $0,7.5$ and $15 \%$ bran, from the two DDD systems. 


\section{0 / Chapter 32}

deviation was calculated to be $0.0030 \mathrm{~g} \mathrm{~cm}^{-3}$, intermediate between the two values reported above. The inclusion of bran appears to increase the variability between replicate results. Figure 3(b) also suggests that adding $7.5 \%$ bran had little effect on the minimum density achieved, while adding $15 \%$ bran increased the minimum density, implying a decreased degree of expansion. Figure 3(a) reveals the reason for this; in both samples containing $15 \%$ bran, gas retention was suddenly lost after about 26 minutes. This is an example of a hiccup as described above, which was not seen so clearly in the subsequent trials described below. This illustrates that the occurrence of the points of weakness that give rise to these hiccups is a probabilistic event in which the dough formulation affects the probability that such a weakness will occur. In the case of doughs containing $15 \%$ bran, the physical presence of the large and mechanically rigid bran particles puncturing gas bubbles and the sample surface and interfering with the gluten network increases the likelihood of retaining points of weakness, despite using the swirling procedure of sampling protocol 3. The doughs with bran were stickier, in agreement with reports from other workers (Lai et al., 1989a), making it more difficult to avoid creating these points of weakness during sampling.

\subsection{Effects of mixer scale and bran level}

Further trials were carried out in which the minimum dough density and baked loaf volume were measured, in duplicate, for doughs containing 0, 7.5 and $15 \%$ bran, and mixed in three scales of geometrically similar laboratory mixer, the MDD10, MDD50 and MDD125. The water absorption for doughs without bran was $61.8 \%$, increasing to $64.8 \%$ with $7.5 \%$ bran and $69.6 \%$ with $15 \%$ bran, while the optimum work input decreased correspondingly from 12.1 to 10.7 and $10.9 \mathrm{Wh} \mathrm{kg}^{-1}$.

Figure 4 shows the loaf volumes, weights and specific loaf volumes obtained at each bran level for each of the three mixers. Clearly the addition of bran increased loaf weights; the extra water added during mixing was retained in the loaves, in agreement with previous work (Dreese and Hoseney, 1982; Rao and Rao, 1991). Loaf volumes and specific loaf volumes decreased as bran was added, in agreement with most previous work (Shetlar and Lyman, 1944; Pomeranz et al., 1977; Pomeranz, 1977; Dubois, 1978; Shogren et al., 1981; Dreese and Hoseney, 1982; Lai et al., 1989a-c; Gan et al., 1989, 1992; Zhang and Moore, 1999; Nelles et al., 1998; de Kock et al., 1999). Specific loaf volumes were smaller for loaves from doughs prepared in the smaller scales of mixer, probably because the relatively larger surface area allowed greater losses of gases during the early stages of baking, prior to heat-setting of the loaf structure, and hence gave lower oven spring. Table 1 presents the texture, volume and baking scores, which were clearly depressed by the addition of bran, but were generally comparable for the loaves from the two larger scales of mixer. The results also confirmed that the MDD10 system is capable of producing acceptable loaves from dough formulations containing bran. Figure 5 shows cross sections of loaves from each of the three mixers at each level of bran substitution.

Figure 6 shows the minimum densities obtained for each bran level and mixer, averaged over the two replicates. The pooled standard deviation was calculated to be $0.014 \mathrm{~g} \mathrm{~cm}^{-3}$, somewhat larger than obtained previously, primarily due to an unrepresentatively large discrepancy between the two replicates from the MDD50 at 7.5\% bran; other replicate data points from the two density meters agreed much more closely. Excluding these two data points gave a standard deviation of $0.0086 \mathrm{~g} \mathrm{~cm}^{-3}$, more in line with previous estimates; however, the former value has been used to indicate error bars on the figure. Two-way ANOVA indicated that bran level had a significant effect on the minimum density $(\mathrm{P}<0.04)$, but mixer scale did not $(\mathrm{P}=0.16)$ (despite the, evidently insignificantly, larger values of minimum density obtained from the MDD50 with bran). Inspection of the data suggests that adding $7.5 \%$ bran had little effect on the minimum density obtained, but that adding $15 \%$ bran significantly reduced the expansion capacity of the dough during proving, in agreement with the preliminary results reported above. However these results do not correlate directly with the baking results, which indicated a decrease in loaf volume at $7.5 \%$ bran and a further decrease at $15 \%$. Figure 7 shows the baked loaf specific volume versus the specific volume 
corresponding to the minimum density $\left(=1 / \rho_{\min }\right)$; the former are higher, due to oven spring, and display a much wider range, indicating that much of the effect of bran and mixer scale is manifest during baking.
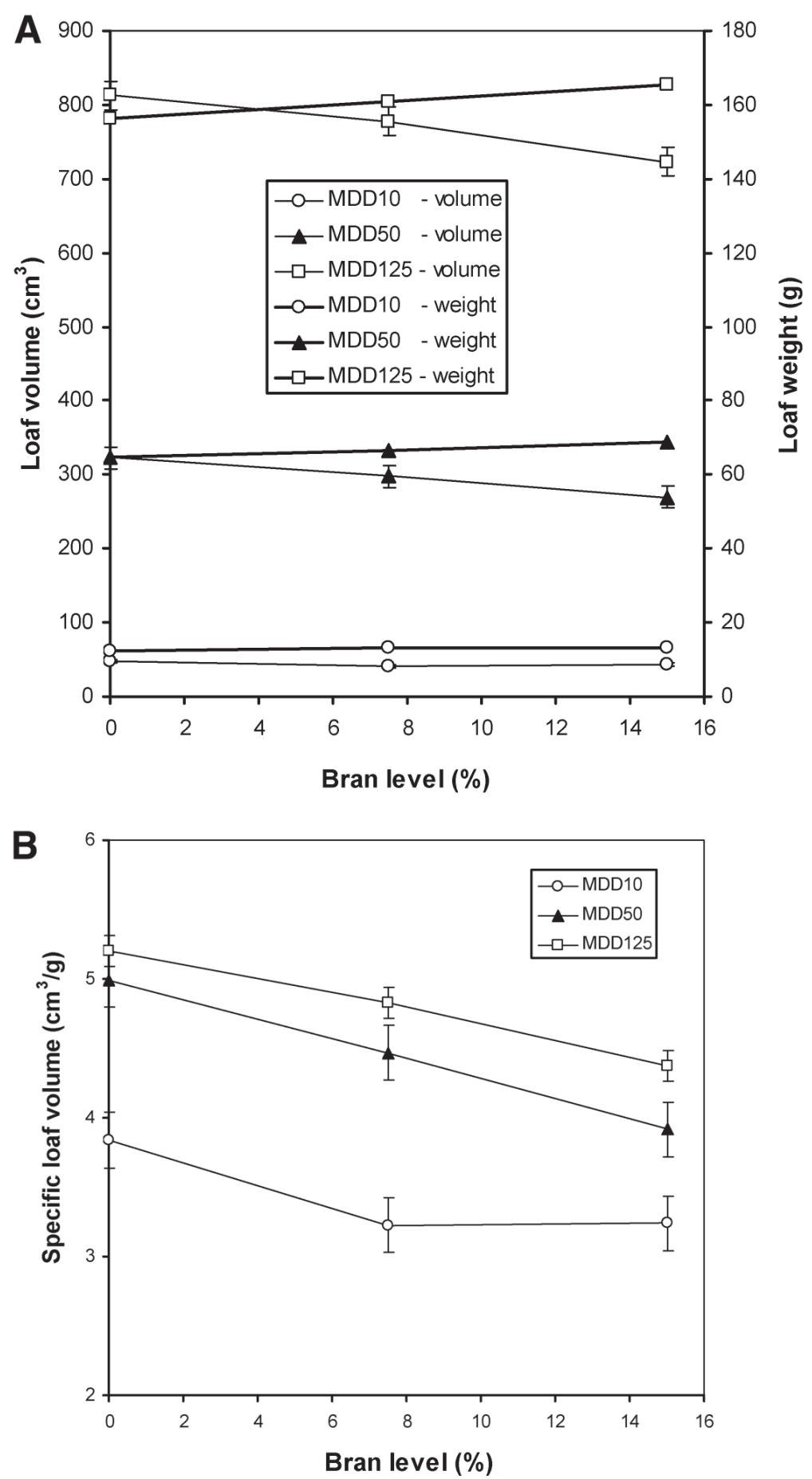

Figure 4. Effect of mixer scale and bran level on (a) loaf volumes and weights; and (b) specific loaf volumes. 


\section{2 / Chapter 32}

Table 1. Texture, volume and baking scores for loaves prepared with different levels of bran and mixed in three scales of mixer. Values are averages of four assessments. Baking scores were not calculated for the MDD10. Differences in texture or baking score of 1 are considered significant.

\begin{tabular}{lcccc}
\hline & Volume $\left(\mathbf{c m}^{3}\right)$ & Texture (1-11) & Baking score \\
\hline MDD10 & 0\% bran & $48 \pm 2^{\mathrm{a}}$ & 10.25 & - \\
& $7.5 \%$ bran & $42 \pm 2$ & 4.25 & - \\
15\% bran & $44 \pm 2$ & 2.75 & \\
MDD50 & & & 24.5 \\
& 0\% bran & $323 \pm 11$ & 8.5 & 17.5 \\
& 7.5\% bran & $298 \pm 11$ & 5.5 & 11.5 \\
15\% bran & $270 \pm 11$ & 4.25 & 25.5 \\
& & & 18.5 \\
& & & 10 & 14 \\
\hline
\end{tabular}

${ }^{a}$ Based on a pooled standard deviation from duplicate loaves.

The action of bran in depressing loaf volumes is multi-faceted, with numerous contributing factors. The results presented here indicate that at least part of the effect, particularly at higher bran levels, occurs during proving. Most workers investigating the effects of bran on bread have only measured final baked loaf volumes, and have not distinguished effects of bran at the proving stage of breadmaking. Dreese and Hoseney (1982) presented evidence that the effect of bran in depressing loaf volumes occurred during baking; the current work supports this suggestion, but also indicates that at high levels the effect of bran is in part evident during proving. The precise nature of the effect of bran on gas retention during proving will be the subject of further studies, with a view to alleviating the deleterious effects of bran on bread quality.

The work presented here also indicates the potential of the DDD technique for exploring the actions and efficacy of various bakery ingredients, in particular those that manifest their effects during proving. The DDD approach is similar in some ways to some commercial instruments such as the Chopin Rheofermentometer, but is quicker than this instrument and yields results that are more readily interpretable in terms of gas bubble dynamics and mass transfer of $\mathrm{CO}_{2}$ during proving (Chiotellis and Campbell, 2003a,b).

\section{Conclusions}

The DDD technique can accurately determine the maximum expansion capacity of a dough during proving, which relates to the volume of the final baked loaf. It is potentially a rapid test of ingredient functionality and the effects of dough formulation and process variables. The effect of bran in breadmaking is at least in part evident during proving, with high levels of bran compromising the gas retention capacity of the dough. The three scales of the laboratory dough mixer design used give doughs with comparable maximum expansion capacities during proving, although differences in specific loaf volume become evident during baking.

\section{Acknowledgements}

This work was carried out during a sabbatical visit by GMC to Crop \& Food Research. GMC is grateful to UMIST for time and funding for the sabbatical and to the UK's Biotechnology and Biological Sciences Research Council (BBSRC) for further funding under its ISIS Long Term Travel Award Scheme. He is also grateful to Crop \& Food Research staff for hosting his visit, in particular Marco Morgenstern, Nigel Larsen and Tim Lindley. The authors are grateful to others 
Bubbles in Food II: Novelty, Health, and Luxury / 333

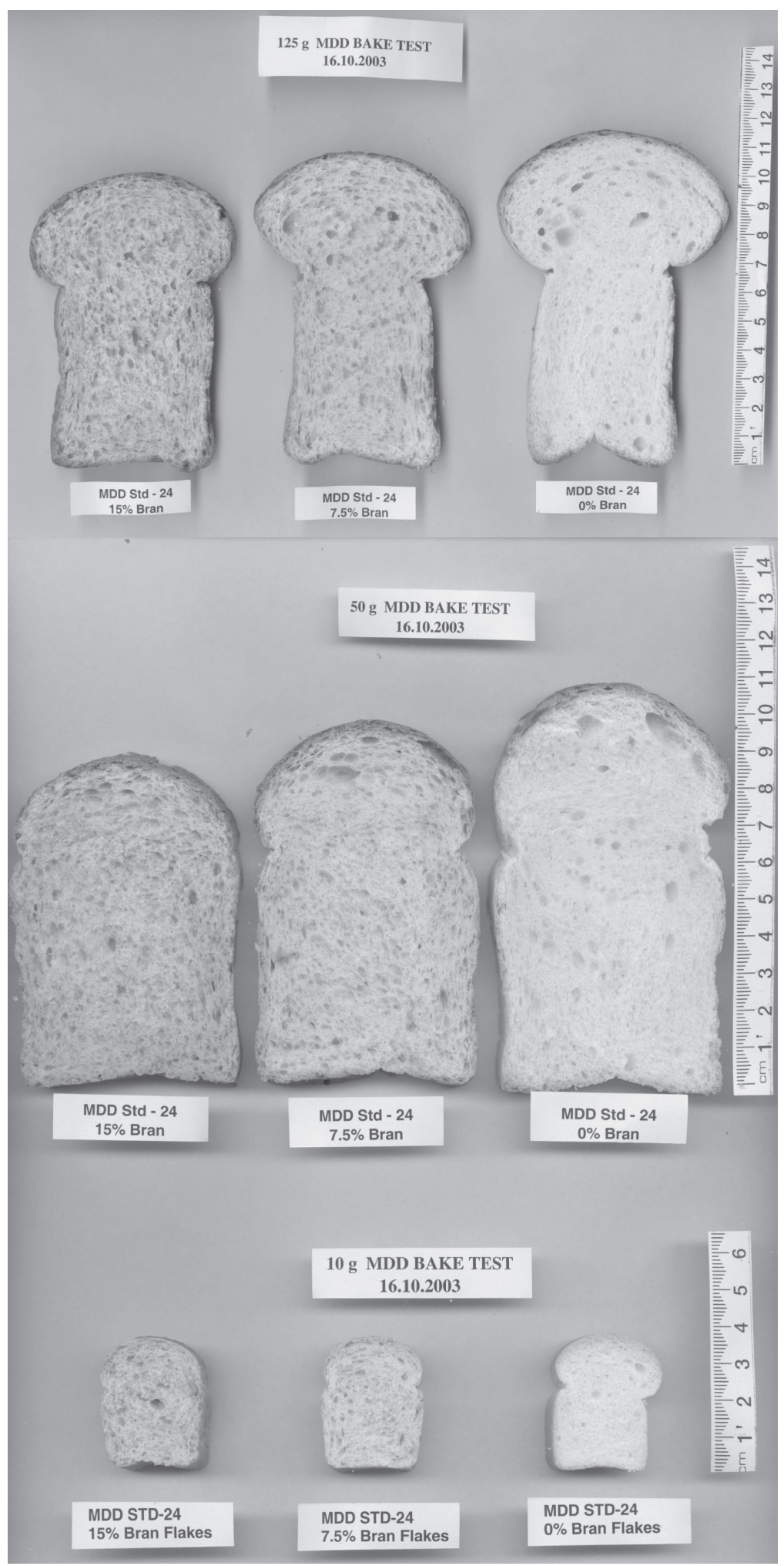

Figure 5. Cross sections of loaves prepared in the MDD125, MDD50 and MDD10 with 0, 7.5 and $15 \%$ bran substitution. 


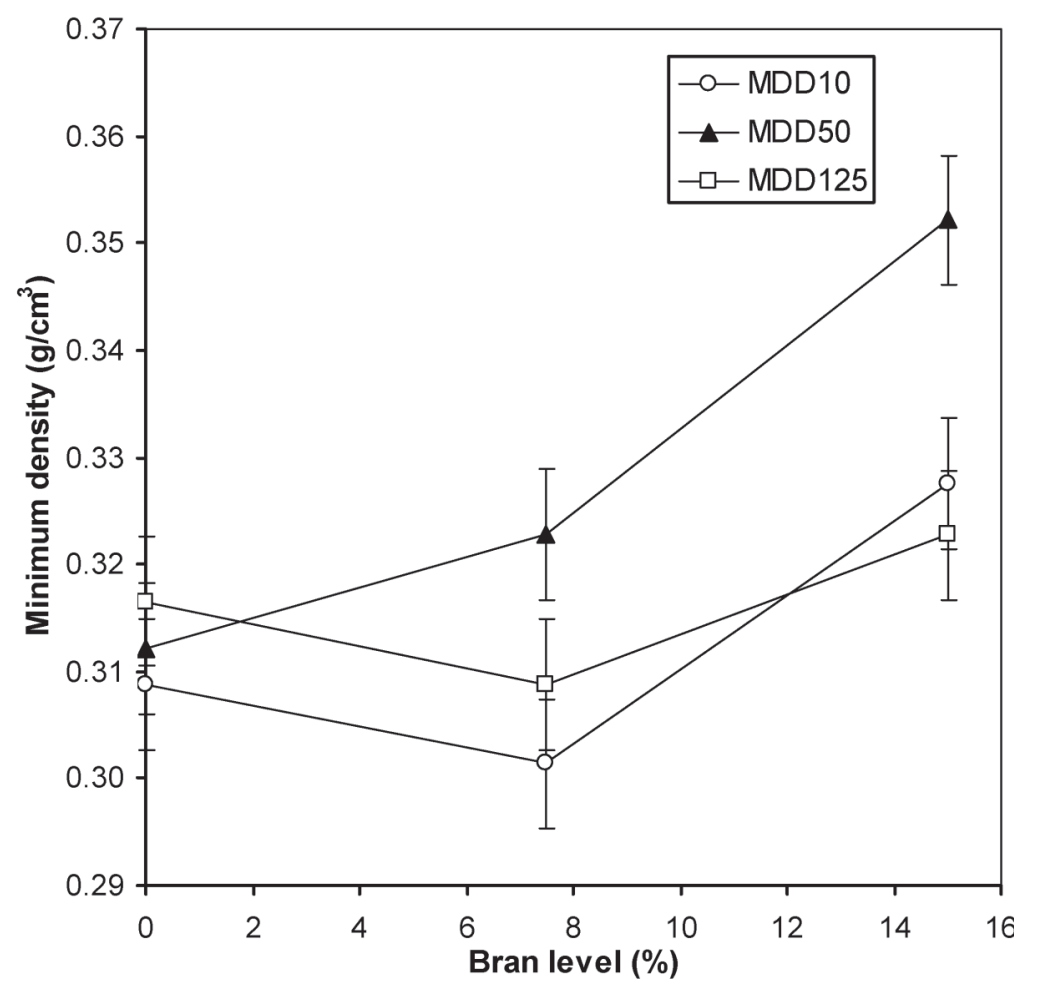

Figure 6. Effect of mixer scale and bran level on maximum expansion capacity (minimum density).

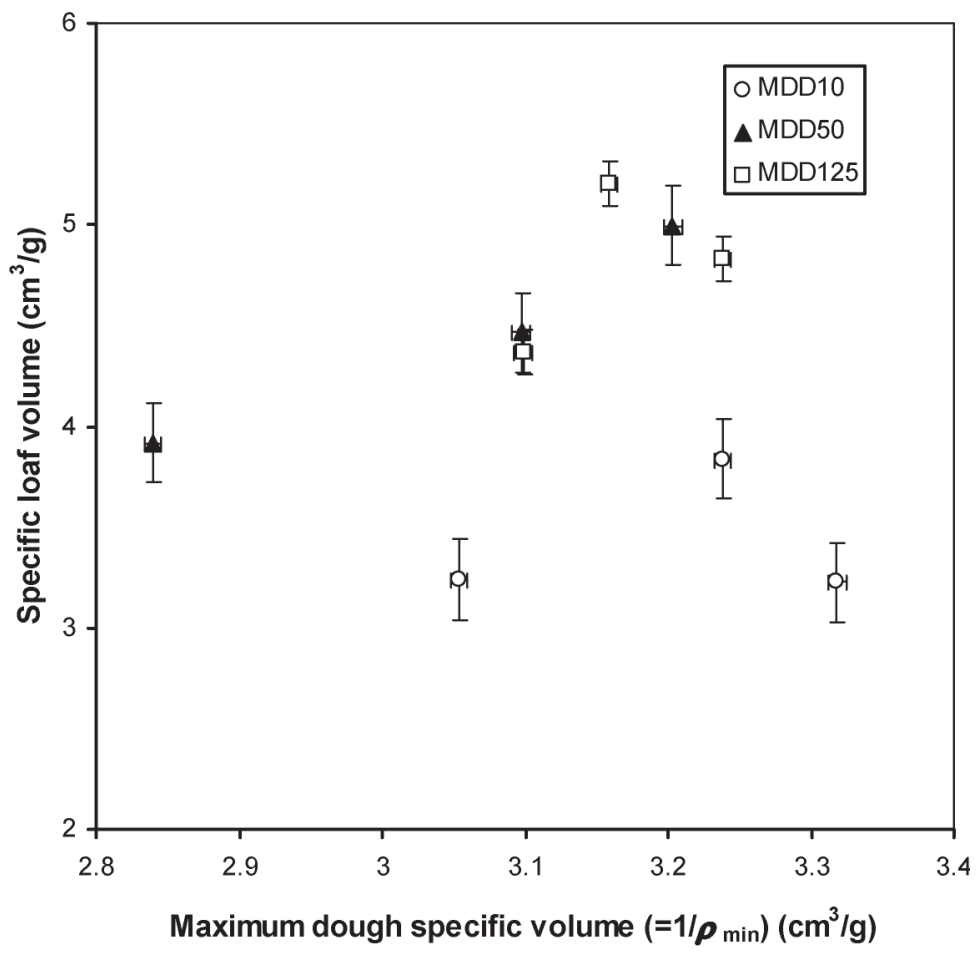

Figure 7. Baked loaf specific volumes versus specific volumes of doughs at the point of minimum density. 
at Crop \& Food Research for assistance in building the two DDD systems and for other technical assistance and useful discussions, in particular Arran Wilson, Tom Finger, Jafar Al-Hakkak, Shiva Rao, Marcus Newberry and Lyall Simmons. The Satake Corporation of Japan is gratefully acknowledged for its support of the activities of the Satake Centre for Grain Process Engineering.

\section{References}

Békés F. and Wrigley C.W. (2002). Efficient testing of wheat quality at the milligram or megagram level. Pages 101-112 in Wheat Quality Elucidation: The Bushuk Legacy. Ng PKW and Wrigley CW (Eds.), American Association of Cereal Chemists, St. Paul, Minnesota, USA.

Campbell G.M., Herrero-Sanchez R., Payo-Rodriguez R. and Merchan, M.L. (2001). Measurement of dynamic dough density, and the effect of surfactants and flour type on aeration during mixing and gas retention during proving. Cereal Chem. 78:272-277.

Chamberlain N., Collins T.H. and Elton, G.A.H. (1967). The Chorleywood Bread Process: Effect of rate of dough mixing. FMBRA Report No. 1, March.

Chiotellis E. and Campbell G.M. (2003a). Proving of bread dough I: Modelling the evolution of the bubble size distribution. Trans IChemE Part C: Food Bioprod. Proc. 81:194-206.

Chiotellis E. and Campbell G.M. (2003b). Proving of bread dough II: Measurement of gas production and retention. Trans IChemE Part C: Food Bioprod. Proc. 81,:207-216.

de Kock S., Taylor J. and Taylor J.R.N. (1999). Effect of heat treatment and particle size of different brans on loaf volume of brown bread. Lebensmittel-Wissenschaft und-Technologie. 32:349-356.

Dobraszczyk B.J. and Morgenstern M.P. (2003). Review: Rheology and the breadmaking process. J. Cereal Sci. 38(3): 229-245.

Dreese PC. and Hoseney R.C. (1982). Baking properties of the bran fraction from brewer's spent grains. Cereal Chem. 59:89-91.

Dubois D.K. (1978). The practical application of fiber materials in bread production. Bakers Digest 5(2):30-33.

Frazier P.J., Daniels N.W.R. and Eggitt P.W.R. (1975). Rheology and the continuous breadmaking process. Cereal Chem. 52:106r-130r.

Gan Z., Ellis P.R., Vaughan J.G. and Galliard T. (1989). Some effects of non-endosperm components of wheat and of added gluten on wholemeal bread microstructure. J. Cereal Sci. 10:81-91.

Gan Z., Galliard T., Ellis P.R., Angold R.E. and Vaughan J.G. (1992). Effect of the outer bran layers on the loaf volume of wheat bread. J. Cereal Sci. 15:151-163.

Gras P.W. and Békés F. (1996). Small-scale dough testing: the development of instrumentation and application as a research tool. Pages 507-510 in Gluten '96, Proceedings of the 6th International Gluten Workshop, Wrigley C.W. (Ed.), Royal Australian Chemical Institute, Melbourne, Australia.

He H. and Hoseney R.C. (1991). Gas retention of different cereal flours. Cereal Chem. 68(4):334-336.

Heaps P.W., Webb T., Eggitt P.W.R. and Coppock J.B.M. (1967). Studies on the mechanical factors affecting dough development. J. Food Technol. 2:37-43.

Kilborn R.H. and Tipples K.H. (1972). Factors affecting mechanical dough development. I. Effect of mixing intensity and work input. Cereal Chem. 49:34-47.

Lai C.S., Hoseney R.C. and Davis A.B. (1989a). Effects of wheat bran in breadmaking. Cereal Chem. 66:217-219.

Lai C.S., Hoseney R.C. and Davis A.B. (1989b). Functional effects of shorts in breadmaking. Cereal Chem. 66:220-223.

Lai C.S., Davis A.B. and Hoseney R.C. (1989c). Production of whole wheat bread with good loaf volume. Cereal Chem. 66, 224-227.

Martin P.J., Chin N.L., Campbell G.M. and Morrant C.F. (2004). Aeration during bread dough mixing III. Effect of scale-up. Trans IChemE Part C: Food Bioprod. Proc. 82:282-290.

Nelles E.M., Randall P.G. and Taylor J.R.N. (1998). Improvement of brown bread quality by prehydration treatment and cultivar selection of bran. Cereal Chem. 75:536-540.

Pomeranz Y., Shogren M.D., Finney K.F. and Bechtel D.B. (1977). Fiber in breadmaking-effects on functional properties. Cereal Chem. 54(1):25-41.

Pomeranz Y. (1977) Fiber in breadmaking - A review of recent studies. Bakers Digest 51(5):94-96, 142.

Rao P.H. and Rao H.M. (1991). Effect of incorporating wheat bran on the rheological characteristics and bread making quality of flour. J. Food Sci. Technol. 28:92-97. 


\section{6 / Chapter 32}

Shetlar M.R. and Lyman J.F. (1944). Effect of bran on bread baking. Cereal Chem. 21:295-304.

Shogren M.D., Pomeranz Y. and Finney K.F. (1981). Counteracting the deleterious effects of fiber in breadmaking. Cereal Chem. 58(2):142-144.

Skeggs P.K. and Kingswood K.K., (1981). Mechanical dough development - Pilot scale studies. Cereal Chem. 58:256-260.

Sosulski F.W. and Wu K.K. (1988). High-fiber breads containing field pea hulls, wheat, corn and wild oat brans. Cereal Chem. 65:186-191.

Wilson A.J., Wooding A.R. and Morgenstern M.P. (1997). Comparison of work input requirement on laboratoryscale and industrial-scale mechanical dough development mixers. Cereal Chem. 74:715-721.

Wilson A.J., Morgenstern M.P. and Kavale S. (2001). Mixing response of a variable speed 125 g laboratory scale mechanical dough development mixer. J. Cereal Sci. 34:151-158.

Wooding A.R. (1997). Agricultural strategies to reduce dough mixing requirements. PhD thesis, The University of Sydney, NSW, Australia.

Wootton M. and Sham-Ud-Din M. (1986). The effects of aqueous extraction on the performance of wheat bran in bread. J. Sci. Food Agric. 37:387-390.

Zhang D. and Moore W.R. (1997). Effect of wheat bran particle size on dough rheological properties. J. Sci. Food Agric. 74:490-496.

Zhang D. and Moore W.R. (1999). Wheat bran particle size effects on bread baking performance and quality. J. Sci. Food Agric. 79:805-809. 